American Journal of Agricultural and Biological Sciences 5 (1): 107-113, 2010

ISSN 1557-4989

(C) 2010 Science Publications

\title{
Multienvironment Analysis of Traits Relation and Hybrids Comparison of Maize Based on the Genotype by Trait Biplot
}

\author{
${ }^{1}$ Saeed Safari Dolatabad, ${ }^{2}$ Rajab Choukan, ${ }^{3}$ Eslam Majidi Hervan and ${ }^{4}$ Hamid Dehghani \\ ${ }^{1}$ Department of Plant Breeding, Sciences and Research Branch, \\ Islamic Azad University (IAU), Tehran, Iran \\ ${ }^{2}$ Seed and Plant Improvement Institute, Karaj, Iran \\ ${ }^{3}$ Department of Plant Breeding, Sciences and Research Branch, \\ Islamic Azad University (IAU), Tehran, Iran \\ ${ }^{4}$ Department of Plant Breeding, Faculty of Agriculture, \\ The University of Tarbiat Modarres, Tehran, Iran
}

\begin{abstract}
Problem statement: The Genotype by Trait (GT) biplot can be used to compare cultivars on the basis of multiple traits and to identify cultivars that are particularly good in certain traits and therefore can be candidates for parents in plant breeding program. Approach: The objective of this study was to evaluate the maize hybrids based on Genotype by Traits (GT) biplot to examine its usefulness in visualizing maize trait relationship and its application in hybrids comparison. Results: Correlation coefficient between phenological-agronomic traits showed that there is a strong positive relationship between all of the measured traits, except Anthesis-Silking Interval (ASI). This pattern approximately repeated during three years. Also, correlation coefficient between grain yield components reveals a positive or negative relation between measured traits. These results reflect the complexity of the correlation among the grain yield components. The genotype by trait comparison indicated that KSC 700 had greater value for rows number per ear and kernel depth whereas OSSK 602 and ZP 684 had greater value for thousand kernel weight and grain number per row. Conclusion: Results indicate that the pattern of the polygon view traits varied across three years. Consequently, GT biplot describes the interrelationships among traits and it was used to identifying hybrids that are good for some particular traits.
\end{abstract}

Key words: Zea mays L., GGE biplot, Genotype $\times$ Environment, genotype by trait biplot, traits correlation

\section{INTRODUCTION}

Genotype $\times$ Environment $(\mathrm{GE})$ interaction is commonly observed by crop producers and breeders as a differential ranking of genotype yields among locations and years. Plant breeders conduct MultipleEnvironment Trials (MET) primarily to identify the superior cultivar and secondarily to determine if the target region can be subdivided into different mega environments (Yan et al., 2000). The GE interaction results from the differential responses of genotypes across a range of environments (Allard and Bradshaw, 1964; Kang, 1998; 2004). The GE interaction reduces the correlation between phenotypic and genotypic values (Comstock and Moll, 1963) and complicates the selection of the best genotypes (Ebdon and Gauch, 2002; Magari and Kang, 1993).
The GGE biplot, which is composed of two factors, effect of Genotype $(\mathrm{G})$ and Genotype by Environment interaction (GE) and must be considered simultaneously, in cultivar evaluation (Yan and Tinker, 2005; Yan et al., 2000). The GGE biplot methodology was used to visually analyze the results of Sites Regression (SREG) analysis of MET data. This method uses a biplot to show the two factors (G plus GE) that are important in genotype evaluation and that are also the sources of variation in SREG model analysis of MET data (Yan et al., 2001).

The GGE biplot has been used to identify high yielding and adapted cultivars by many researchers such as Fan et al. (2007) and Setimela et al. (2007) for maize, Yan et al. (2000) and Morris et al. (2004) for wheat, Samonte et al. (2005) for rice, Dehghani et al.

Corresponding Author: Saeed Safari Dolatabad, Department of Plant Breeding, Sciences and Research Branch, Islamic Azad University (IAU), Tehran, Iran 
(2006) and Yan and Tinker (2005) for barley, Sabaghnia et al. (2006) for lentils and Kang et al. (2006) for common bean.

Furthermore, superior crop cultivars must be evaluated on the basis of multiple traits to ensure that the selected cultivars have acceptable performance in variable environments within the target region (Yan and Rajcan, 2002; Yan and Tinker, 2005).

The Genotype by Trait (GT) biplot can be used to compare cultivars on the basis of multiple traits and to identify cultivars that are particularly good in certain traits and therefore can be candidates for parents in plant breeding program. The polygon view of GT biplot allows visualization of the which-won-where pattern that identifies genotypes that are best for certain traits and the traits vector allows visualization of the interrelationship among traits and it is also used as independent selection criteria based on several traits (Yan and Rajcan, 2002).

Iran is the world's largest importers of agricultural products, importing about $30 \%$ of its needs. Maize is an important field crop in the agricultural system in Iran. One way to increase maize production is to grow hybrids best adapted to different environments and growing conditions. To evaluate performances of maize hybrids, multi environment trials are frequently used in Iran. The objective of this study was to evaluate the hybrids based on Genotype by Traits (GT) biplot, to examine its usefulness in visualizing maize trait relationship and its application in hybrid comparison.

\section{MATERIALS AND METHODS}

Experimental locations and maize hybrids: Data used in this study were obtained from the national maize multi-environment yield trials. These trials were conducted in three years (2006-2008) by the Seed and Plant Improvement Institute in Iran. Each year, 14 maize hybrids (Table 1) were grown and tested in 9 field stations (Table 2) in different regions in Iran. These stations were the Moghan (MGN) field station in the northwestern, Karaj (KRJ) field station in the northern, Esfahan (ESF) field station in the center, Shiraz (two sowing dates, SHZ A and SHZ B) field station, Darab (DRB) field station and Kerman (KRM) field station in the southern, Khoramabad (KHM) and Kermanshah (KSH) field stations in the western and Dezfol (two sowing dates, DZF A and DZF B) field station in the southwestern part of the country. The name of the hybrids, their code and their origins are given in Table 1.
Experimental design and culture: In each location a randomized complete block design with four replicates was used. Plots consisting of two rows $\left(9 \mathrm{~m}^{2}\right.$ with row spacing at $75 \mathrm{~cm}$ and plant spacing at $35 \mathrm{~cm}$ ) were hand planted and harvested. Plots were initially over-planted and later thinned to two plants per hill. Plant density is 76000 plants $\mathrm{ha}^{-1}$ in all environments. Irrigation system was similar for all experimental locations and they were irrigated once per week. Nitrogen $\left(200 \mathrm{~kg} \mathrm{ha}^{-1}\right.$ before sowing and $200 \mathrm{~kg} \mathrm{ha}^{-1}$ after thinning) and phosphorus (300 $\mathrm{kg} \mathrm{ha}^{-1}$ before sowing) fertilizers were applied according to the recommendations of the Soil and Water Research Institute for each location.

Data collection: Several phenological traits such as days to tasseling (days between emergence and mid tasseling), days to anthesis (days between emergence and mid pollination), days to maturity (days between emergence and black layer) and anthesis-silking interval (difference between days to mid silking and days to mid pollination), agronomic traits (plant height and ear height), grain yield components (thousand kernel weight, grain number per row, kernel depth and row number per ear) were recorded for all locations and years. In order to obtain appropriate results, these traits were divided into two groups (phenological-agronomic traits and grain yield components) and were analyzed, separately.

Table 1: Names, codes, and origin of 14 maize hybrids

\begin{tabular}{llll}
\hline Name of hybrids & Hybrids code & FAO group & Origin of hybrids \\
\hline ZP 677 & G1 & 600 & Serbia \\
ZP 434 & G2 & 400 & Serbia \\
ZP 684 & G3 & 600 & Serbia \\
BC 572 & G4 & 500 & Croatia \\
BC 678 & G5 & 670 & Croatia \\
BC 666 & G6 & 660 & Croatia \\
BC 582 & G7 & 580 & Croatia \\
BC 5982 & G8 & 510 & Croatia \\
BC 682 & G9 & 680 & Croatia \\
OSSK 602 & G10 & 600 & Croatia \\
G-3261 & G11 & 500 & Greece \\
ZP 599 & G12 & 500 & Serbia \\
KSC 700 & G13 & 700 & Iran \\
KSC 704 & G14 & 700 & Iran \\
\hline
\end{tabular}

Table 2: Geographic coordinates of test locations

\begin{tabular}{llll}
\hline Location & Longitude & Latitude & Evaluation $(\mathrm{m})$ \\
\hline Karaj (KRJ) & $51^{\circ} 00^{\prime}$ & $35^{\circ} 49^{\prime}$ & 1360 \\
Esfahan (ESF) & $51^{\circ} 39^{\prime}$ & $32^{\circ} 38^{\prime}$ & 1575 \\
Moghan (MGN) & $48^{\circ} 03^{\prime}$ & $39^{\circ} 01^{\prime}$ & 1100 \\
Dezfol (DZF) & $48^{\circ} 24^{\prime}$ & $32^{\circ} 24^{\prime}$ & 143 \\
Shiraz (SHZ) & $52^{\circ} 33^{\prime}$ & $29^{\circ} 36^{\prime}$ & 1491 \\
Darab (DRB) & $54^{\circ} 34^{\prime}$ & $28^{\circ} 46^{\prime}$ & 1150 \\
Kerman (KRM) & $57^{\circ} 05^{\prime}$ & $30^{\circ} 17^{\prime}$ & 1748 \\
Kermanshah (KSH) & $47^{\circ} 07^{\prime}$ & $34^{\circ} 19^{\prime}$ & 1322 \\
Khoramabad (KHM) & $48^{\circ} 21^{\prime}$ & $33^{\circ} 32^{\prime}$ & 1171 \\
\hline
\end{tabular}


The genotype by trait biplot: The Genotype by Trait (GT) biplot approach (Yan and Rajcan, 2002) was used to display the genotype by trait data in a biplot and is based on the following formula:

$$
\frac{\alpha_{i j}-\beta_{j}}{\sigma_{j}}=\sum_{n=1}^{2} \lambda_{n} \xi_{i n} \eta_{j n}+\varepsilon_{i j}=\sum_{n=1}^{2} \xi_{i n}^{*} \eta_{j n}^{*}+\varepsilon_{i j}
$$

Where:

$\alpha_{i j}=$ The mean value of genotype $\mathrm{i}$ for trait $\mathrm{j}$

$\beta_{\mathrm{j}}=$ The mean value of all genotypes for trait $\mathrm{j}$

$\sigma_{\mathrm{j}}=$ The standard deviation of trait $\mathrm{j}$ among genotype means

$\lambda_{\mathrm{n}}=$ The singular value for Principal Component (PCn)

$\xi_{\text {in }}=$ The PCn score for genotype $\mathrm{i}$

$\eta_{\mathrm{jn}}=$ The PCn score for trait $\mathrm{j}$

$\varepsilon_{\mathrm{ij}}=$ The residual associated with genotype $\mathrm{i}$ in trait $\mathrm{j}$

To achieve trait-focused scaling between genotype and trait scores the singular value $\lambda_{\mathrm{n}}$ has to be absorbed by the singular vector for genotype $\xi_{\text {in }}$ and for traits $\eta_{\mathrm{jn}}$. That is, $\xi_{\mathrm{in}}^{*}=\xi_{\mathrm{in}} \lambda_{\mathrm{n}}^{0}=\xi_{\mathrm{in}}$ and $\eta_{\mathrm{jn}}^{*}=\eta_{\mathrm{jn}} \lambda_{\mathrm{n}}^{1}=\eta_{\mathrm{jn}} \lambda_{\mathrm{n}}$. Because $\mathrm{n}=2$ in a biplot, only PC1 and PC2 are retained in the model and such a model tends to be best for extracting patterns and rejecting noise from the data. A Genotype by Trait (GT) biplot is constructed by plotting PC1 scores against PC2 scores for each genotype and each trait (Yan and Rajcan, 2002).

All analyses reported in this study were conducted by using the GGE biplot software (Yan, 2001; Yan and Kang, 2003) (www.ggebiplot.com).

\section{RESULTS}

Trait relations: The GT biplot reveals the interrelationships between traits and it is also used as independent selection criteria based on several traits and in yield trials for grain yield evaluation (Yan and Rajcan, 2002). The correlation coefficient between any two traits is approximated by the cosine of the angle between their vectors. Two traits are positively correlated if the angle between their vectors is $<90^{\circ}$, negatively correlated if the angle is $>90^{\circ}$, independent if the angle is $90^{\circ}$.

The GT biplot for each of the three years explained high proportion of the total variation of the data. Provided that the biplot explained a high amount of the total variation, the correlation coefficient between each two traits is approximated by the cosine of the angle between the vectors (Yan and Rajcan, 2002). Phenological traits such as days to Tasseling (TSL), days to anthesis (PLD), Days to Maturity (DMA) and Anthesis-Silking Interval (ASI) and agronomic traits such as plant Height (HGT) and ear Height (HGX) were analyzed. The correlation coefficient between each two traits calculated and examined by the cosine of the angle between the vectors (Fig. 1).

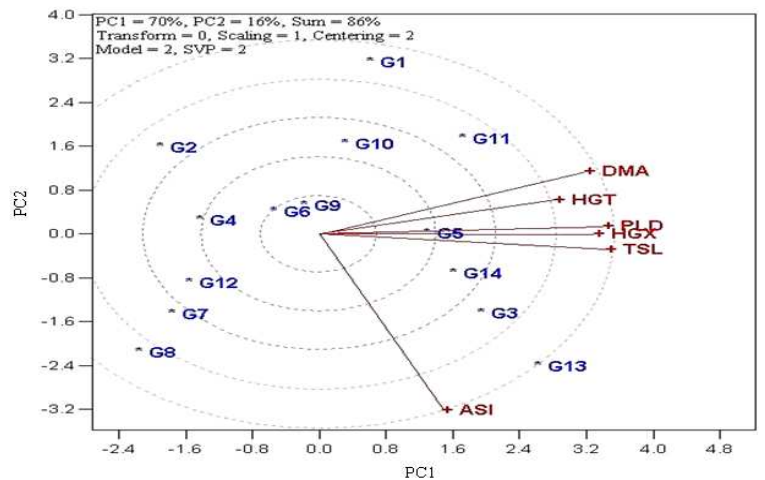

(A)

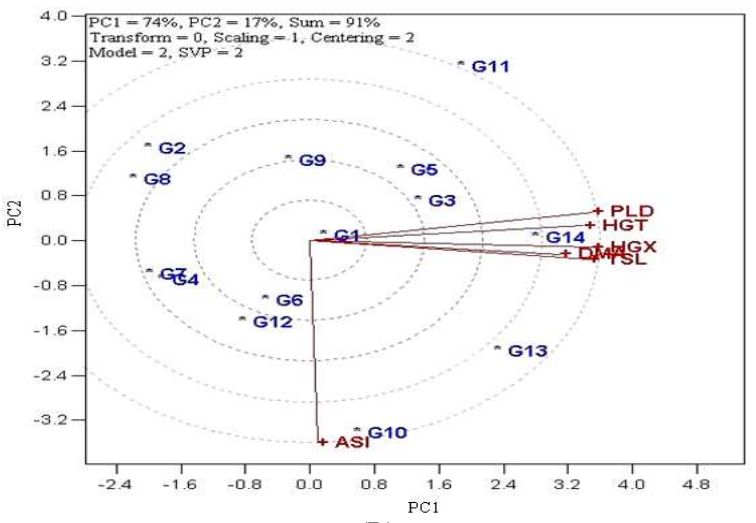

(B)

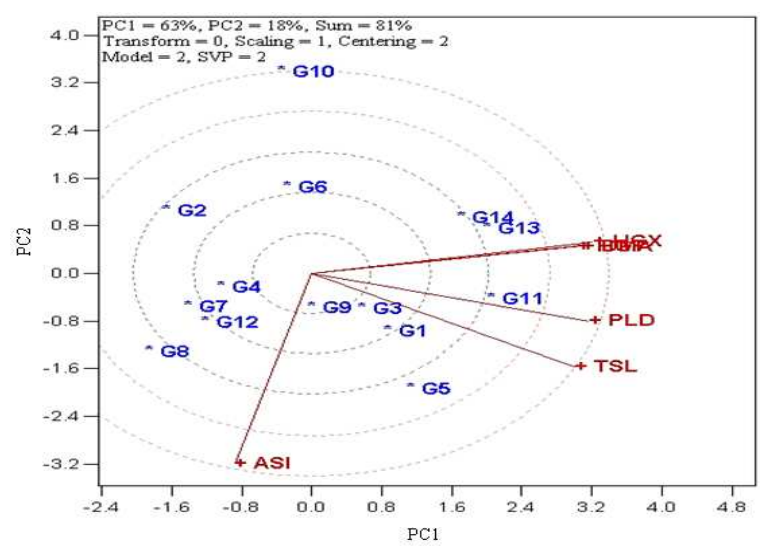

(C)

Fig. 1: The yearly correlation between different traits: (A) 2006; (B) 2007; (C) 2008. Days to Maturity (DMA), days to Tasseling (TSL), days to anthesis (PLD), Anthesis-Silking Interval (ASI), plant Height (HGT) and ear Height (HGX) 
Therefore, there is a strong positive relationship between all of the measured traits, except AnthesisSilking Interval (ASI) as indicated by the small acute angles between their vectors (Fig. 1). This pattern approximately was repeated during 3 years.

Also, grain yield components (Thousand Kernel Weight (TKW), grain number per row (SNO), kernel depth (SDP) and Row Number per ear (RNO)) were analyzed separately. The GT biplot for each of the three years explained 77,82 and $84 \%$ of the total variation of the standardized data (Fig. 2). Therefore, according to these results, can be fundamental patterns among the traits which were captured by the GT biplots. The most prominent relations showed by these biplots are: (i) a close correlation between row number per ear and kernel depth (Fig. 2A and B) as indicated by the near perpendicular vectors, (ii) a positive relation between thousand kernel weight and grain number per row (Fig. 2A and C) and between kernel depth and grain number per row (Fig. 2B) in addition between thousand kernel weight and kernel depth (Fig. 2C), (iii) a negative association between row number per ear and grain number per row (Fig. 2A and C) and between row number per ear and thousand kernel weight (Fig. 2A and $2 \mathrm{~B}$ ). These results reflect the complexity of the correlation among the measured traits.

Hybrids comparison: The Genotype by Trait (GT) biplot is used to identify genotypes that are best for certain traits. The GT biplot is also used as independent selection criteria based on several traits and in yield trials for grain yield evaluation (Yan and Rajcan, 2002). Fig. 3 is a GT biplot with a polygon view that presents the data of 14 maize hybrids on three years with four traits. The vertex hybrids for the first year (Fig. 3A) are G13, G10, G3, G2, G7 and G8 and the traits fell into the sectors of G13 and G10 hybrids. Therefore, it seems that G13 hybrid had the highest values of kernel depth (SDP) and Rows Number per ear (RNO); G10 hybrid had the highest values of Thousand Kernel Weight $(\mathrm{TKW})$ and grain number per row (SNO). Figure 3B indicates that G13 was highest in rows number per ear and G11 was highest in kernel depth and grain number per row whereas G3 had the highest values in thousand kernel weight. Also, the third year (Fig. 3C) showed that G3 was best for grain number per row and G1 and G10 were best for kernel depth whereas G8 had greater value for rows number per ear.

Based on the three years data (Fig. 3D), it is resulted that G13 had greater value for rows number per ear and kernel depth whereas G3 were best for thousand kernel weight and grain number per row. No traits fell into sectors with G2, G7 and G8 hybrids as the vertices, indicating that they had not great values for the expression of any of the concerned traits. These results indicate that the pattern of the polygon view varied across years (Fig. 3).

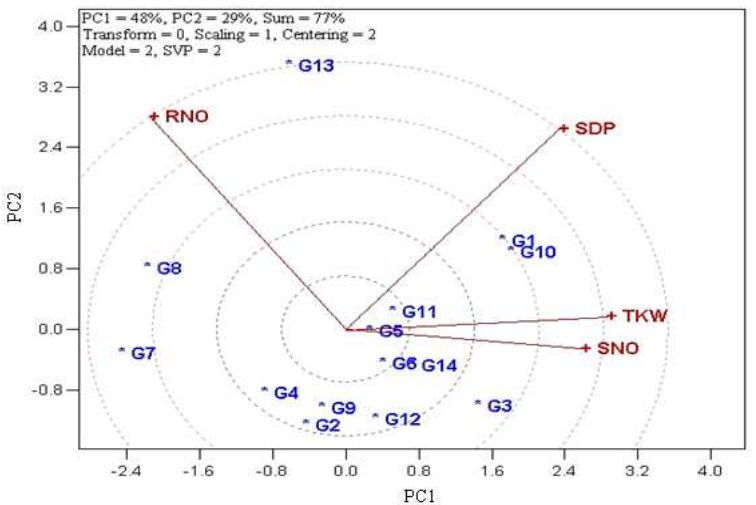

(A)

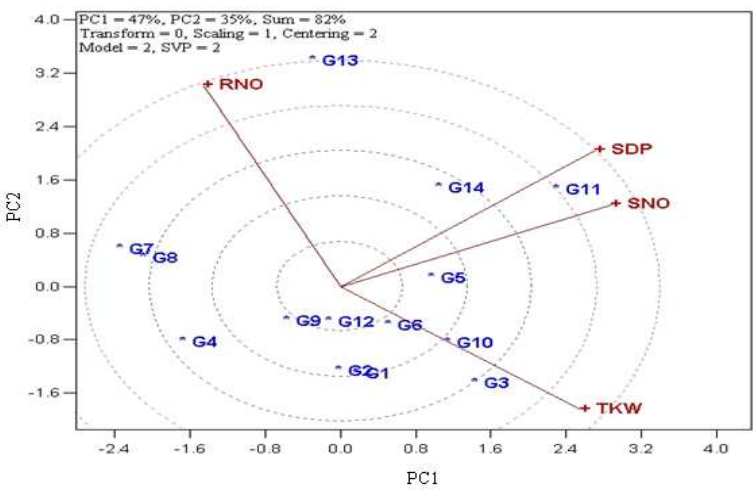

(B)

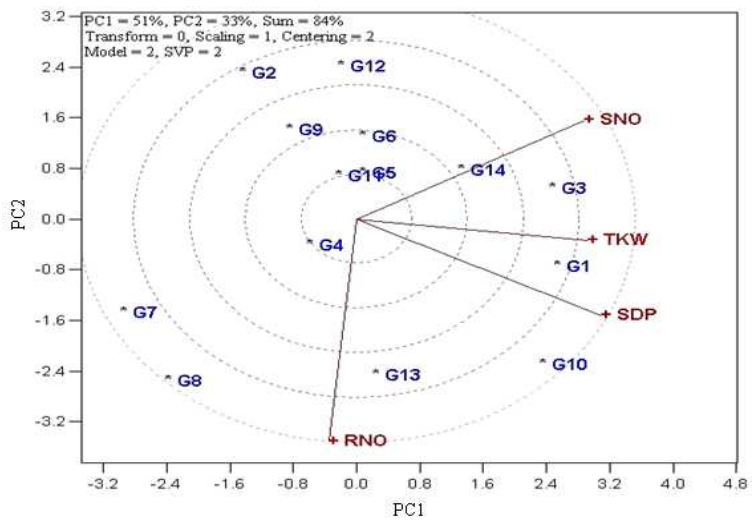

(C)

Fig. 2: The yearly correlation between different traits: (A) 2006; (B) 2007; (C) 2008. Thousand kernel weight (TKW), grain number per row (SNO), kernel depth (SDP) and Rows Number per ear (RNO) 
Am. J. Agri. \& Biol. Sci., 5 (1): 107-113, 2010

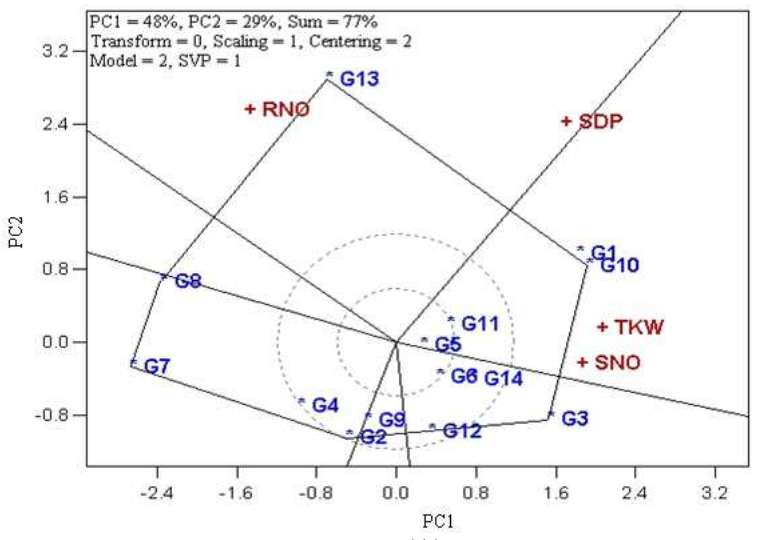

(A)

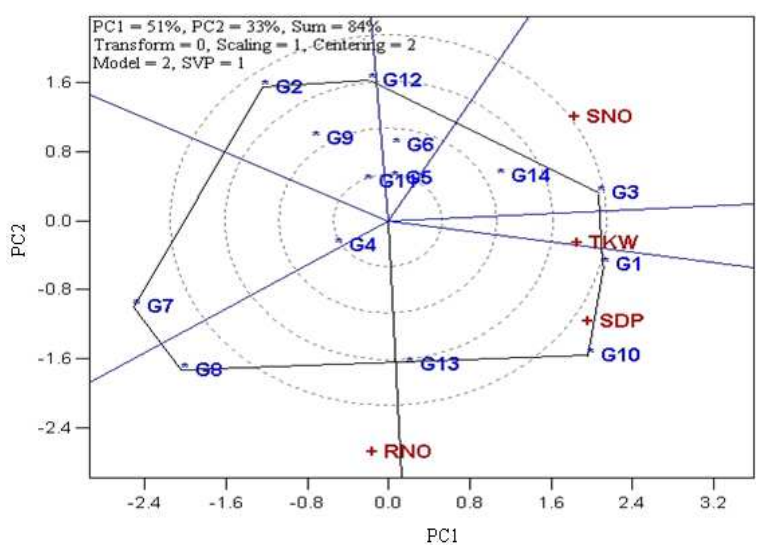

(C)

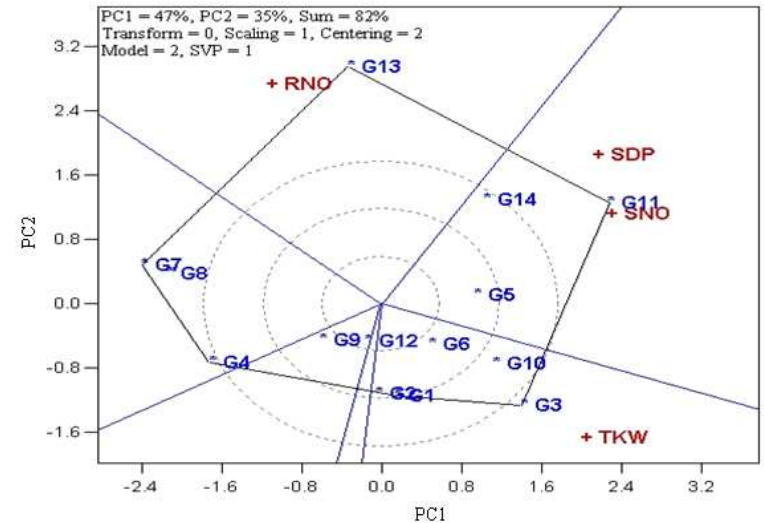

(B)

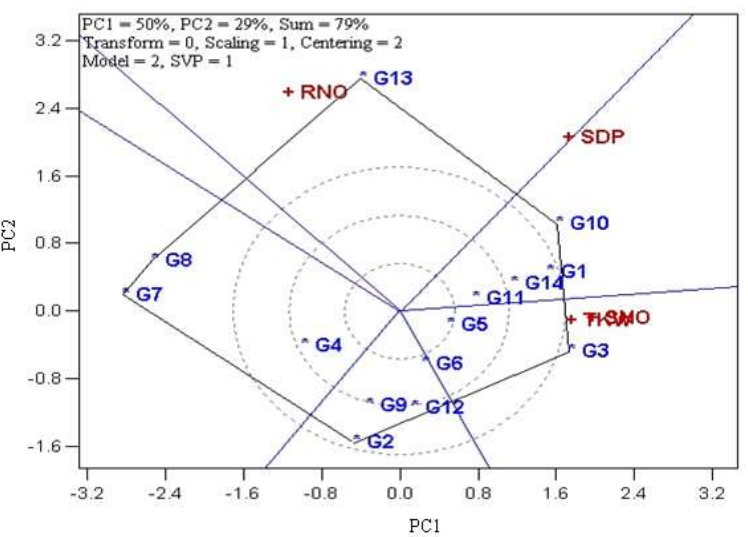

(D)

Fig. 3: The polygon view of the hybrid by traits. (A) 2006; (B) 2007; (C) 2008; (D) three years. Thousand Kernel Weight (TKW), grain number per row (SNO), kernel depth (SDP) and Rows Number per ear (RNO)

\section{DISCUSSION}

Genotype by Trait (GT) biplot is effective tool for revealing the interrelationships among the maize traits and it provides a tool for visual comparison among genotypes on the basis of multiple traits. Also, it can be used in independent culling based on multiple traits and in comparing selection strategies (Yan and Rajcan, 2002).

Many researchers such as Shakoor et al. (2007); Chapman and Edmeades (1999); Turi et al. (2007) and Andrea et al. (2006) examined the relation between different traits of maize. As an example, Shakoor et al. (2007) reported the relation between ear height and plant height and between days $50 \%$ silking and plant height are positive and the relation between ear height and ears plant ${ }^{-1}$ is negative, as well as in this present study it has been resulted that the relation between plant Height (HGT) and ear Height (HGX) is positive (Fig. 1) and the between grain number per row (SNO) and Rows Number per ear (RNO) is negative (Fig. 2). These results are relatively similar to the reports mentioned above. In this study the method of calculation to find the relation between traits in GT biplot is different from simple correlation coefficient because the GT biplot approach is constructed by the first two principal components (PC1 and PC2), so in this approach, the total yield variation could not be explained (Fig. 1 and 2). In addition, GT biplot graphically describes the interrelationships among all measured traits on the basis of overall pattern of the data in different environments and years, whereas simple correlation coefficients only describe the relationships between two traits (Yan and Rajcan, 2002). Therefore, simple correlation between traits does not agree with those of relation between traits in GT biplot completely. Also, GT biplot can be used to visualize the relation among traits (breeding objectives) 
which facilitates a systems understanding of the crop and facilitates identification of traits that can be used in indirect selection for a target trait (Yan and Tinker, 2005). Considering what mentioned above, interrelationships among measured traits on the basis of overall pattern of the data (GT biplot) are better than the simple correlation coefficients that only describe the relationships between two traits.

Genotype by Trait (GT) biplot provides a tool for visual comparison among genotypes on the basis of multiple traits. Also, it can be used in independent culling based on multiple traits and in comparing selection strategies, which is important for both cultivar evaluation and parent selection (Yan and Rajcan, 2002; Yan and Tinker, 2005). Based on this study for example G1 and G10 were best for Thousand Kernel Weight (TKW) and grain number (SNO) and G13 was best for kernel depth (SDP) and rows number per ear (RNO) witch these traits are favorable characteristics for hybrids (Fig. 3A). Also, Thousand Kernel Weight (TKW), grain number per row (SNO), kernel depth (SDP) and Rows Number per ear (RNO) which are major yield components and greatly affect grain yield should be considered in the maize breeding programs for improvement of these traits and yield. Independent culling based on the traits can also be used to aid genotype selection (Yan and Rajcan, 2002).

\section{CONCLUSION}

Results indicate that the GT biplot for each of the three years explained high proportion of the total variation of the data and the pattern of the polygon view traits varied across three years. Consequently, Provided that the biplot explained a high amount of the total variation, GT biplot describes the interrelationships among traits and it was used to identifying hybrids that are good for some particular traits.

\section{ACKNOWLEDGMENT}

We would like to thank Dr. Khodarahmi, Assistant Professor, Seed and Plant Improvement Institute Karaj, Iran for his technical assistances and performing GGE biplot analysis as well as for his suggestions. The authors would also like to thank Eng. Moeini, maize research officer for his kindly assistance in recording data.

\section{REFERENCES}

Allard, R.W. and A.D. Bradshaw, 1964. Implication of genotype $\times$ environmental interaction in applied plant breeding. Crop Sci., 5: 503-506. DOI: 10.1007/BF00022654
Andrea, K.E., M.E. Otegui, A.G. Cirilo and G. Eyheravide, 2006. Genotypic variability in morphological and physiological traits among maize inbred linesnitrogen responses. Crop Sci., 46: 1266-1276. DOI: 10.2135/cropsci2005.07-0195

Chapman, S.C. and G.O. Edmeades, 1999. Selection improves drought tolerance in tropical maize populations: II direct and correlated responses among secondary traits. Crop Sci., 39: 1315-1324. http://crop.scijournals.org/cgi/content/abstract/39/5 $/ 131$

Comstock, R.E. and P.H. Moll, 1963. Genotypeenvironment interaction. Proceeding of the Symposium on Statistical Genetics and Plant Breeding, National Academy of Sciences, Washington, DC, NAS-NRC Publication, pp: 164196.

Dehghani, H., A. Ebadi and A. Yousefi, 2006. Biplot analysis of genotype by environment interaction for barley yield in Iran. Agron. J., 98: 388-393. DOI: 10.2134/agronj2004.0310

Ebdon, J.S. and H.G. Gauch, 2002. Additive main effect and multiplicative interaction analysis of national turfgrass performance trials: I. Interpretation of Genotype $\times$ Environment interaction. Crop Sci., 42: 489-496.

Fan, X.M., M.S. Kang, H.Y. Zhang, J. Tan and C. Xu, 2007. Yield stability of maize hybrids evaluated in multi-environment trials in Yunnan, China. Agron. J., 99: 220-228. DOI: 10.2134/agronj2006.0144

Kang, M.S., 1998. Using genotype-by-environment interaction for crop cultivar development. Adv. Agron., 62: 199-252.

Kang, M.S., 2004. Breeding: Genotype by Environment Interaction. In: Encyclopedia of Plant and Crop Science, Goodman, R.M. (Ed.). Marcel Dekker, New York, ISBN: 0-4913-3438-6, pp: 218-221.

Kang, M.S., V.D. Aggarwal and R.M. Chirwa, 2006. Adaptability and stability of bean cultivars as determined via yield stability statistic and GGE biplot analysis. J. Crop Improve., 15: 97-120. http://www.informaworld.com/smpp/content conte nt

Magari, R. and M.S. Kang, 1993. Genotype selection via a new yield stability statistic in maize yield trials. Euphytica, 70: 105-111. DOI: 10.1007/BF00029647

Morris, C.F., K.G. Campbell and G.E. King, 2004. Characterization of the end-use quality of soft wheat cultivars from the eastern and western US germplasm "pools”. Plant Genet. Resour., 2: 59-69. http://ggebiplot.com/SoftWheatQuality.pdf 
Sabaghnia, N., H. Dehghani and S.H. Sabaghpour, 2006. Nonparametric methods for interpreting Genotype $\times$ Environment interaction of lentil genotypes. Crop Sci., 46: 1100-1106. http://crop.scijournals.org/cgi/content/abstract/46/3 $/ 110$

Samonte, S.O.P.B., L.T. Wilson, A.M. Mc Clung and J.C. Medley, 2005. Targeting cultivars onto rice growing environments using AMMI and SREG GGE biplot analyses. Crop Sci., 45: 2414-2424. DOI: $10.2135 /$ cropsci2004.0627

Setimela, P.S., B. Vivek, M. Banziger, J. Crossa and F. Maideni, 2007. Evaluation of early to medium maturing open pollinated maize varieties in SADC region using GGE biplot based on the SREG model. Field Crops Res., 103: 161-169. DOI: 10.1016/j.fcr.2007.05.010

Shakoor, M.S., M. Akbar and A. Hossain, 2007. Correlation and path coefficients studies of some morphological traits in maize double crosses. Pak. J. Agric. Sci., 44: 213-216.

Turi, N.A., S.S. Shah, S. Ali, H. Rahman, T. Ali and M. Sajjad, 2007. Genetic variability for yield parameters in maize (Zea Mays L.) genotypes. J. Agric. Biol. Sci., 2: 1-3.

Yan, W., L.A. Hunt, Q. Sheng and Z. Szlavnics, 2000. Cultivar evaluation and mega-environment investigation based on the GGE biplot. Crop Sci., 40: 597-605.

http://crop.scijournals.org/cgi/content/abstract/40/3 1597
Yan, W., 2001. GGE biplot Windows application for graphical analysis of multienvironment trial data and other types of two-way data. Agron. J., 93: 1111-1118.

http://agron.scijournals.org/cgi/content/abstract/93/ 5/1111

Yan, W., P.L. Cornelius, J. Crossa and L.A. Hunt, 2001. Comparison of two types of GGE biplots for studying genotype by environment interaction. Crop Sci., 41: 656-663.

Yan, W. and I. Rajcan, 2002. Biplot analysis of test sites and trait relations of soybean in Ontario. Crop Sci., 42: 11-20.

http://crop.scijournals.org/cgi/content/abstract/42/1 $/ 11$

Yan, W. and M.S. Kang, 2003. GGE Biplot Analysis: A Graphical tool for Breeders, Geneticists and Agronomists. CRC Press, Boca Raton, FL., ISBN: 0-8493-1338-4, pp: 63-88.

Yan, W. and N.A. Tinker, 2005. An integrated system of biplot analysis for displaying, interpreting and exploring genotype by-environment interactions. Crop Sci., 45: 1004-1016. DOI: 10.2135/cropsci2004.0076 